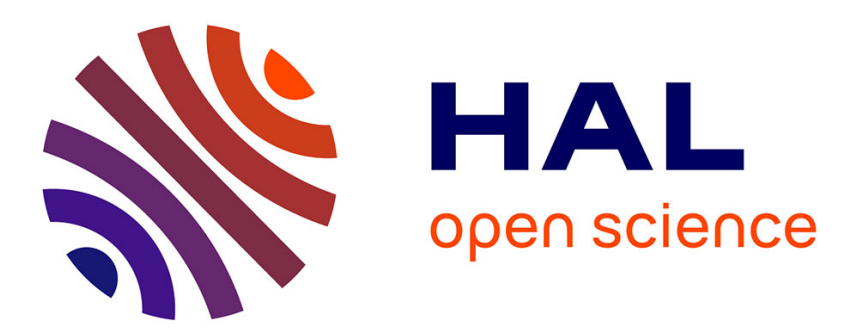

\title{
Simultaneous determination of two coefficients in Itô diffusion processes: theoretical and numerical approaches
}

\author{
Michel Cristofol, L Roques
}

\section{To cite this version:}

Michel Cristofol, L Roques. Simultaneous determination of two coefficients in Itô diffusion processes: theoretical and numerical approaches. Springer. Inverse problems and related topics, 310, Springer, 2018, Springer Proceedings in Mathematics \& Statistics, 978-981-15-1591-0. 10.1007/978-981-151592-7_3 . hal-03560886

\section{HAL Id: hal-03560886 https://hal.science/hal-03560886}

Submitted on 19 Feb 2022

HAL is a multi-disciplinary open access archive for the deposit and dissemination of scientific research documents, whether they are published or not. The documents may come from teaching and research institutions in France or abroad, or from public or private research centers.
L'archive ouverte pluridisciplinaire HAL, est destinée au dépôt et à la diffusion de documents scientifiques de niveau recherche, publiés ou non, émanant des établissements d'enseignement et de recherche français ou étrangers, des laboratoires publics ou privés.

$$
\text { Copyright }
$$




\title{
Simultaneous determination of two coefficients in Itô diffusion processes: theoretical and numerical approaches
}

M. Cristofol and L. Roques

\begin{abstract}
In this paper, we consider a one-dimensional Itô diffusion process $X_{t}$ with possibly nonlinear drift and diffusion coefficients. In a first part, we show that both coefficients are simultaneously uniquely determined by the observation of the expectation and variance of the process, during a small time interval, and starting from any values $X_{0}$ in a given subset of $\mathbb{R}$. Then in a second part, we present some numerical simulations which illustrate that this type of observation can be used in practice to estimate the coefficients of a diffusion process.
\end{abstract}

\section{Introduction}

The following results are partially based on a recent work by the same authors [12]. We are interested with a one-dimensional Itô diffusion process $X_{t} \in \mathbb{R}$ satisfying stochastic differential equations of the form:

$$
d X_{t}=b\left(X_{t}\right) d t+\sigma\left(X_{t}\right) d W_{t}, t \in[0, T] ; X_{0}=x,
$$

where $T>0, W_{t}$ is the one-dimensional Wiener process and $b: \mathbb{R} \rightarrow \mathbb{R}, \sigma: \mathbb{R} \rightarrow$ $\mathbb{R}, \sigma>0$, are Lipschitz-continuous functions. Under these assumptions, the solution of the equation (1) is unique in the sense of Theorem 5.2.1 in [18]. The term $b\left(X_{t}\right) d t$

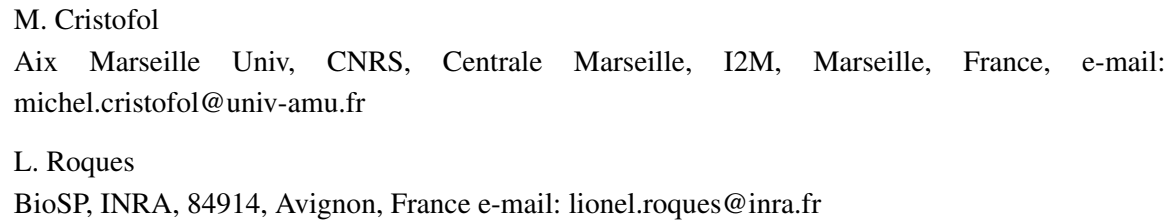


can be interpreted as the deterministic part of the equation, while $\sigma\left(X_{t}\right) d W_{t}$ is the stochastic part of the equation. In the sequel, the functions $b$ and $\sigma$ are called the drift term and diffusion term, respectively.

These equations arise in several domains of applications, such as biology, physics or financial mathematics with non-constant coefficients $\alpha, \beta$, to model stock prices in the Black-Scholes model. The reconstruction of unknown parameters in stochastic differential equations has been largely addressed recently. We can refer among others to $[1,2,16,17]$ with different analytic strategies and to [22] for a survey on the topic, this list being far from to be exhaustive.

The aim of our study is to determine the drift term $b$ and the diffusion term $\sigma$ for general equations of the form (1), based on observations of the stochastic process $X_{t}$. Equivalently, this means showing the uniqueness of the coefficients $b$ and $\sigma$ that correspond to a given observation. The main type of observation that we consider is the expectation $E^{x}\left[f\left(X_{t}\right)\right]=E\left[f\left(X_{t}\right) \mid X_{0}=x\right]$, of some function of the stochastic process $X_{t}$, for instance a momentum if $f(s)=s^{k}$ for some $k \geq 0$. The observation is carried out during a small time interval and for initial conditions $X_{0}$ in a small neighborhood of a given $x_{0} \in \mathbb{R}$. In that respect we use parabolic partial differential equation (PDE) technics inspired from the theory of inverse problems.

The Itô diffusion processes are related to PDEs by the Kolmogorov's backward theorem (see e.g. theorem 8.1.1 in [18]). Consider a function

$$
f \in C^{2}(\mathbb{R}) \text { such that }|f(x)| \leq C e^{\delta x^{2}},
$$

for $\delta>0$ small enough and some $C>0$. Define

$$
u(t, x)=E^{x}\left[f\left(X_{t}\right)\right]=E\left[f\left(X_{t}\right) \mid X_{0}=x\right],
$$

where $X_{t}$ is the solution of (1) with $X_{0}=x$. The Kolmogorov's backward theorem implies that $u$ is the unique solution in $C_{1}^{2}\left(\mathbb{R}_{+} \times \mathbb{R}\right)$ of:

$$
\partial_{t} u=\frac{1}{2} \sigma^{2}(x) \partial_{x x} u+b(x) \partial_{x} u, t \geq 0 ; u(0, x)=f(x) .
$$

For parabolic equations of the form (4), several inverse problems have already been investigated. In all cases, the main question is to show the uniqueness of some coefficients in the equation, based on exact observations of the solution $u(t, x)$, for $(t, x)$ in a given observation region $\mathscr{O} \subset[0,+\infty) \times \mathbb{R}$. Furthermore, one of the most challenging goal is to obtain such uniqueness results using the smallest possible observation region. 
Most uniqueness results in inverse problems for parabolic PDEs have been obtained using the method of Carleman estimates [4] on bounded domains. This method requires, among other measurements, knowledge of the solution $u(\tau, x)$ at some time $\tau>0$ and for all $x$ in the domain [3, 10, 11, 14, 23, 24]. Other approaches are based on a semi-group formulation of the solutions, but use the same type of observations of the solution on the whole domain, at a given time [5]. More recent approaches $[7,19,20,21]$ lead to uniqueness results for one or several coefficients, under the assumption that $u$ and its first spatial derivative are known at a single point $x_{0}$ of a bounded domain, and for all $t$ in a small interval $(0, \varepsilon)$, and that the initial data $u(0, x)$ is known over the entire domain. On the other hand, the case of unbounded domains is less addressed (see [8]).

Here, contrarily to most existing approaches:

- the domain is unbounded. All of the results in [7, 19, 20, 21] require a bounded domain assumption. Most of the studies based on other methods (e.g., Carleman estimates) also assume that the domain is bounded; see [8] or [9] for some results on unbounded domains, in the case of parabolic and hyperbolic operators;

- we determine simultaneously two coefficients in front of a second and a first order term in the PDE. The reconstruction of several coefficients, including a coefficient in front of a second order term, is very challenging not only from the theoretical point of view, but also from the numerical one [13, 6]. Up to our knowledge, theoretical studies on the determination of the diffusion and the drift coefficients from localized observations have not been proposed before;

- our results are interpreted in terms of nonlinear stochastic diffusion processes;

- as in the above-mentioned studies [7, 19, 20,21], we assume that the observation set is reduced to a neighborhood of single point $x_{0}$, during a small time interval.

Our manuscript is organized as follows. In Section 2 we detail our assumptions on the unknown coefficients, on the observations and we state our main results. In Section 3 we present some numerical simulations which illustrate that the type of observation that we use in our theoretical results can be used in practice to estimate the coefficients of a diffusion process. Lastly, the results are proved in Section 4. 


\section{Assumptions and main results}

\subsection{Observations.}

We consider one main type of observations. Let $\varepsilon \in(0, T)$ and $\omega$ an open and nonempty subset in $\mathbb{R}$. The observation sets are of the form

$$
\mathscr{O}_{k}\left[X_{t}\right]=\left\{E^{x}\left[\left(X_{t}\right)^{k}\right] \text {, for } t \in(0, \varepsilon) \text { and } x \in \omega\right\},
$$

for $k=1,2$. We assume $\varepsilon>0$ and $\omega$ can be chosen as small as we want. For the sake of simplicity, and with a slight abuse of notation, for two processes $X$ and $\tilde{X}$, we say that $\mathscr{O}_{k}\left[X_{t}\right]=\mathscr{O}_{k}\left[\tilde{X}_{t}\right]$ if and only if $E^{x}\left[\left(X_{t}\right)^{k}\right]=E^{x}\left[\left(\tilde{X}_{t}\right)^{k}\right]$ for $k=1,2$, for all $t \in(0, \varepsilon)$ and $x \in \omega$.

\subsection{Unknown functions.}

We assume that the unknown functions belong to the function space:

$$
\mathscr{M}:=\{\psi \text { is Lipschitz-continuous and piecewise analytic in } \mathbb{R}\}
$$

A continuous function $\psi$ is called piecewise analytic if there exist $n \geq 1$ and an increasing sequence $\left(\kappa_{j}\right)_{j \in \mathbb{Z}}$ such that $\lim _{j \rightarrow-\infty} \kappa_{j}=-\infty, \lim _{j \rightarrow+\infty} \kappa_{j}=+\infty, \kappa_{j+1}-\kappa_{j}>\delta$ for some $\delta>0$, and $\psi(x)=\sum_{j \in \mathbb{Z}} \chi_{\left[\kappa_{j}, \kappa_{j+1}\right)}(x) \varphi_{j}(x)$, for all $x \in \mathbb{R} ;$

here $\varphi_{j}$ are some analytic functions defined on the intervals $\left[\kappa_{j}, \kappa_{j+1}\right]$, and $\chi_{\left[\kappa_{j}, \kappa_{j+1}\right)}$ are the characteristic functions of the intervals $\left[\kappa_{j}, \kappa_{j+1}\right)$ for $j \in \mathbb{Z}$.

In practice, the assumption $\psi \in \mathscr{M}$ is not very restrictive. For instance, the set of piecewise linear functions in $\mathbb{R}$ is a subset of $\mathscr{M}$.

\subsection{Main results.}

Determining several coefficients of parabolic PDEs is generally far more involved than determining a single coefficient. It requires more and well-chosen observations. 
For instance, four coefficients of a Lotka-Volterra system of parabolic equations have been determined in [21], based on the observation of the solution, starting with three different initial conditions. See also $[5,11]$ with different methods. Here, our result shows that, if the first momentum (expected value) and the second momentum of $X_{t}$ are observed during a small time interval and for $X_{0}=x$ in a small set $\omega \subset \mathbb{R}$, then both coefficients $b$ and $\sigma$ in (1) are uniquely determined.

Theorem 1. Let $b, \tilde{b}, \sigma, \tilde{\sigma} \in \mathscr{M}$. Consider $X_{t}, \tilde{X}_{t}$ the solutions of (1) and of $d \tilde{X}_{t}=$ $\tilde{b}\left(\tilde{X}_{t}\right) d t+\tilde{\sigma}\left(\tilde{X}_{t}\right) d W_{t}, t \in[0, T] ; \tilde{X}_{0}=x$, respectively. Assume that $\mathscr{O}_{k}\left[X_{t}\right]=\mathscr{O}_{k}\left[\tilde{X}_{t}\right]$ for $k=1,2$. Then, $b \equiv \tilde{b}$ and $\sigma \equiv \tilde{\sigma}$ in $\mathbb{R}$.

An immediate corollary of Theorem 1 is that $b$ and $\sigma$ are uniquely determined by the observation of the mean and variance $V^{x}\left[X_{t}\right]=E^{x}\left[X_{t}^{2}\right]-\left(E^{x}\left[X_{t}\right]\right)^{2}$ of the process $X_{t}$ during a small time interval and for $X_{0}=x$ in a small set $\omega \subset \mathbb{R}$. More precisely, define the set

$$
\mathscr{O}_{v}\left[X_{t}\right]=\left\{V^{x}\left[X_{t}\right], \text { for } t \in(0, \varepsilon) \text { and } x \in \omega\right\},
$$

we have the following result.

Corollary 1. Let $b, \tilde{b}, \sigma, \tilde{\sigma} \in \mathscr{M}$. Consider $X_{t}, \tilde{X}_{t}$ the solutions of (1) and $d \tilde{X}_{t}=$ $\tilde{b}\left(\tilde{X}_{t}\right) d t+\tilde{\sigma}\left(\tilde{X}_{t}\right) d W_{t}, t \in[0, T] ; \tilde{X}_{0}=x$, respectively. Assume that $\mathscr{O}_{1}\left[X_{t}\right]=\mathscr{O}_{1}\left[\tilde{X}_{t}\right]$ and $\mathscr{O}_{v}\left[X_{t}\right]=\mathscr{O}_{v}\left[\tilde{X}_{t}\right]$. Then, $b \equiv \tilde{b}$ and $\sigma \equiv \tilde{\sigma}$ in $\mathbb{R}$.

Remark 1. Our result remains true if the observation (5) is replaced by punctual observations at a given point $x_{0} \in \mathbb{R}$ instead of observations in a subdomain $\omega$. More precisely, if (5) is replaced by

$$
\mathscr{O}_{k}^{\prime}\left[X_{t}\right]=\left\{E^{x_{0}}\left[\left(X_{t}\right)^{k}\right], \partial_{x} E^{x}\left[\left(X_{t}\right)^{k}\right]_{\mid x=x_{0}} \text {, for } t \in(0, \varepsilon) \text { and } x \in \omega\right\},
$$

for $k=1,2$, the result of our theorem and corollary can still be obtained, by using the Hopf's Lemma in addition to the strong parabolic maximum principle.

\section{Numerical simulations}

We have shown that non-constant and possibly nonlinear drift and diffusion coefficients of a one-dimensional Itô diffusion process can be determined, based on observations of expectations of some functionals of the process, during a small time interval, and starting any from values $X_{0}=x_{0}$ in a given small subset of $\mathbb{R}$. These results are based on ideal observations, in the sense that they assume that expecta- 
tions are observed (and not only sample paths), and that these observations are not noisy.

The objective of this section is to illustrate that the type of observations in Corollary 1 can be used in practice to estimate the coefficients $b$ and $\sigma$ in

$$
d X_{t}=b\left(X_{t}\right) d t+\sigma\left(X_{t}\right) d W_{t}, t \in[0, T] ; X_{0}=x .
$$

We recall that these observations correspond to the expectation

$$
\mathscr{O}^{1}\left[X_{t}\right]=\left\{E^{x}\left[X_{t}\right], \text { for } t \in(0, \varepsilon) \text { and } x \in \omega\right\},
$$

together with the variance

$$
\mathscr{O}_{v}\left[X_{t}\right]=\left\{V^{x}\left[X_{t}\right] \text {, for } t \in(0, \varepsilon) \text { and } x \in \omega\right\} .
$$

Generation of the observations. We fix two functions $b$ and $\sigma$ in some finitedimensional subspaces $\mathscr{M}_{1}, \mathscr{M}_{2} \subset \mathscr{M}$. For any fixed $x \in \mathbb{R}$ and $t \in(0, \varepsilon)$ we estimate $E^{x}\left[X_{t}\right]$ and $V^{x}\left[X_{t}\right]$ by simulating $N=10^{6}$ trajectories $X_{t}^{i}$, for $t \in(0, \varepsilon)$. Namely, setting $\mathbf{X}_{t}^{x}=\left(X_{t}^{1}, \ldots X_{t}^{N}\right)$, we compute the "empirical" mean and variance

$$
\mathscr{E}\left(\mathbf{X}_{t}^{x}\right)=\frac{1}{N} \sum_{i=1}^{N} X_{t}^{i}, \mathscr{V}\left(\mathbf{X}_{t}^{x}\right)=\frac{1}{N} \sum_{i=1}^{N}\left(X_{t}^{i}\right)^{2}-\mathscr{E}^{2}\left(\mathbf{X}_{t}^{x}\right)
$$

at discrete times $t=t_{1}, \ldots, t_{K} \in(0, \varepsilon)$.

In practice, we set

$$
\mathscr{M}_{1}=\left\{p(x)=\alpha_{0}+\alpha_{1} x+\alpha_{2} x^{2}+\alpha_{3} x^{3}, \alpha_{0}, \alpha_{1}, \alpha_{2}, \alpha_{3} \in \mathbb{R}\right\}
$$

and

$$
\mathscr{M}_{2}=\left\{q(x)=0.1+\left|\beta_{0}+\beta_{1} x\right|, \beta_{0}, \beta_{1} \in \mathbb{R}\right\} .
$$

The coefficients $b(x)$ and $\sigma(x)$ are defined by setting $\alpha_{0}=1, \alpha_{1}=1, \alpha_{2}=1, \alpha_{3}=$ -1 and $\beta_{0}=0.5, \beta_{1}=1$. We assume 100 observation times $t_{j}$, regularly spaced in $[0,1](K=100)$.

The simulation of the trajectories $X_{t}^{i}$ is carried out with the simulate function in Matlab Financial Toolbox ${ }^{\circledR}$. The observations are generated with two different initial conditions: $x=0$ and $x=0.1$ (see Remark 1 for some comment about punctual observations).

Functional to be minimized (objective function). For any $\tilde{b} \in \mathscr{M}_{1}$ and $\tilde{\sigma} \in \mathscr{M}_{2}$, we estimate $E^{x}\left[\tilde{X}_{t}^{x}\right]$ and $V^{x}\left[\tilde{X}_{t}^{x}\right]$ by simulating $\tilde{N}$ trajectories of 


$$
d \tilde{X}_{t}=\tilde{b}\left(\tilde{X}_{t}\right) d t+\tilde{\sigma}\left(\tilde{X}_{t}\right) d W_{t}, t \in[0, \varepsilon] ; \tilde{X}_{0}=x,
$$

and by computing the associated quantities $\mathscr{E}\left(\tilde{\mathbf{X}}_{t}^{x}\right)$ and $\mathscr{V}\left(\tilde{\mathbf{X}}_{t}^{x}\right)$, with $\tilde{\mathbf{X}}_{t}^{x}=\left(\tilde{X}_{t}^{1}, \ldots \tilde{X}_{t}^{\tilde{N}}\right)$ (with initial condition $x$ ). For each initial condition $X_{0}=\tilde{X}_{0}=x$, the match between the trajectories generated with $(\tilde{b}, \tilde{\sigma})$ and the observations $\mathscr{E}\left(\mathbf{X}_{t}^{x}\right), \mathscr{V}\left(\mathbf{X}_{t}^{x}\right)$ is measured by:

$$
F(\tilde{b}, \tilde{\boldsymbol{\sigma}}, x)=\sum_{j=1}^{k}\left(\mathscr{E}\left(\mathbf{X}_{t_{j}}^{x}\right)-\mathscr{E}\left(\tilde{\mathbf{X}}_{t_{j}}^{x}\right)\right)^{2}+\sum_{j=1}^{k}\left(\mathscr{V}\left(\mathbf{X}_{t_{j}}^{x}\right)-\mathscr{V}\left(\tilde{\mathbf{X}}_{t_{j}}^{x}\right)\right)^{2} .
$$

As the observations correspond to two initial conditions $x=0$ and $x=0.1$, the objective function is:

$$
\operatorname{MS}(\tilde{b}, \tilde{\sigma})=F(\tilde{b}, \tilde{\sigma}, 0)+F(\tilde{b}, \tilde{\sigma}, 0.1) .
$$

Note that this objective function is stochastic, as it depends on the trajectories in $\mathbf{X}_{t}^{x}$. In our computations, $M S(\tilde{b}, \tilde{\sigma})$ is either computed with $\tilde{N}=10^{2}$ or $\tilde{N}=10^{3}$ trajectories.

Estimation algorithm. We check whether the coefficients $b$ and $\sigma$ can be numerically determined by minimizing $M S(\tilde{b}, \tilde{\sigma})$ over $\mathscr{M}_{1} \times \mathscr{M}_{2}$. As the objective function $M S$ is stochastic, the minimization of $M S(\tilde{b}, \tilde{\sigma})$ in $\mathscr{M}_{1} \times \mathscr{M}_{2}$ is a stochastic optimization problem. To solve it, we use a the Matlab built-in simulated annealing algorithm [15] (simulannealbnd function in Matlab Global Optimization Toolbox ${ }^{\circledR}$, with initial temperature $10^{4}$, and at most 500 evaluations of the function $M S$ ).

We first check that the objective function $M S$ tends to be smaller as the estimated coefficients tend to resemble the "true" coefficients $(b, \sigma)$. In that respect, we depicted in Fig. 1, the squared $L^{2}$ distance (in $(-2,2)$ ) between $b$ and $\tilde{b}$ (Fig. 1, a) and between $\sigma$ and $\tilde{\sigma}$ (Fig. 1, b) vs the corresponding value of $M S(\tilde{b}, \tilde{\sigma})$. The crosses corresponds to the (500) computations of $M S$ carried out during the simulated annealing algorithm (with $\tilde{N}=10^{3}$ ). We observe that, in spite of the fact that the objective function $M S$ is stochastic, smaller values of $M S$ are associated with coefficients $(\tilde{b}, \tilde{\sigma})$ which tend to be closer to $(b, \sigma)$. Next, we depict in Fig. 2 the result of the minimization procedure, i.e., the functions $(\tilde{b}, \tilde{\sigma})$ associated with the smallest value of $M S(\tilde{b}, \tilde{\sigma})$ obtained with the simulated annealing algorithm, with either $\tilde{N}=10^{2}$ and $\tilde{N}=10^{3}$. In both cases, the global shape of the coefficients $(b, \sigma)$ is well-estimated. Higher values of $\tilde{N}$ lead to a better accuracy of the estimation 


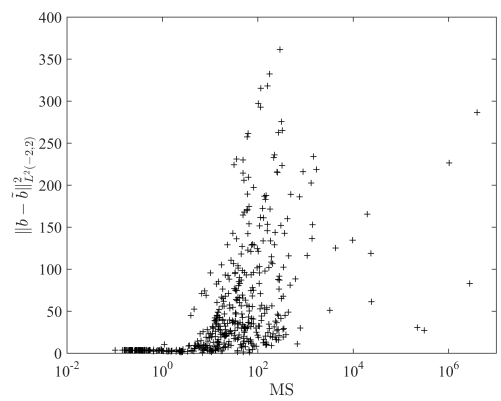

(a)

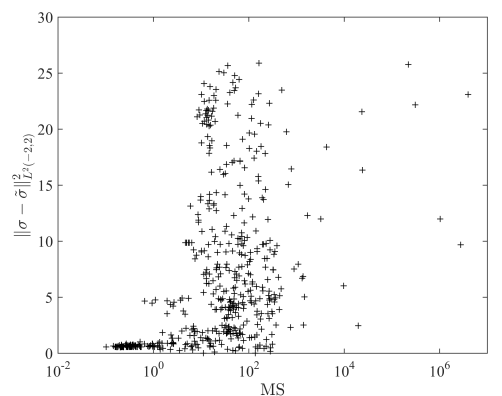

(b)

Fig. 1 (a) Squared $L^{2}$ distance (in $(-2,2)$ ) between $b$ and $\tilde{b}$ vs corresponding value of $M S(\tilde{b}, \tilde{\sigma})$; (b) $L^{2}(-2,2)$ distance between $\sigma$ and $\tilde{\sigma}$ vs corresponding value of $M S(\tilde{b}, \tilde{\sigma})$. Each cross correspond to one evaluation of the function $M S$ carried out by the simulated annealing algorithm (with $\left.\tilde{N}=10^{3}\right)$.



(a)

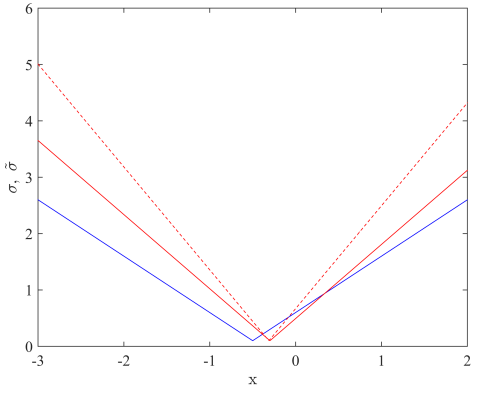

(b)

Fig. 2 (a) blue line: unknown function $b$; dashed red line: function $\tilde{b}$ obtained by minimization of $M S$, with $\tilde{N}=10^{2}\left(\|b-\tilde{b}\|_{L^{2}(-2,2)}^{2}=26.1\right)$; continuous red line: function $\tilde{b}$ obtained by minimization of $M S$, with $\tilde{N}=10^{3}\left(\|b-\tilde{b}\|_{L^{2}(-2,2)}^{2}=3.7\right)$; (b) blue line: unknown function $\sigma$; dashed red line: function $\tilde{\sigma}$ obtained by minimization of $M S$, with $\tilde{N}=10^{2}\left(\|\sigma-\tilde{\sigma}\|_{L^{2}(-2,2)}^{2}=3.7\right)$; continuous red line: function $\tilde{\sigma}$ obtained by minimization of $M S$, with $\tilde{N}=10^{3}\left(\|\sigma-\tilde{\sigma}\|_{L^{2}(-2,2)}^{2}=0.6\right)$.

\section{Proof of Theorem 1}

In this case, we reconstruct simultaneously two coefficients from the principal part and the first order term in equation (4) and this implies to repeat the observations and to consider adapted weight functions in the form (5). We define, for all $t \in[0, T)$ and $x \in \mathbb{R}$, and for $f(s)=s^{k}$, 


$$
\begin{aligned}
& u(t, x)=E^{x}\left[f\left(X_{t}\right)\right]=E\left[f\left(X_{t}\right) \mid X_{0}=x\right], \\
& \tilde{u}(t, x)=E^{x}\left[f\left(\tilde{X}_{t}\right)\right]=E\left[f\left(\tilde{X}_{t}\right) \mid X_{0}=x\right],
\end{aligned}
$$

and $u$ and $\tilde{u}$ are respectively the unique solutions of:

$$
\partial_{t} u=\frac{1}{2} \sigma^{2}(x) \partial_{x x} u+b(x) \partial_{x} u, t \in[0, T), x \in \mathbb{R} ; u(0, x)=f(x),
$$

and

$$
\partial_{t} \tilde{u}=\frac{1}{2} \tilde{\sigma}^{2}(x) \partial_{x x} \tilde{u}+\tilde{b}(x) \partial_{x} \tilde{u}, t \in[0, T), x \in \mathbb{R} \tilde{u}(0, x)=f(x)
$$

Define

$$
B(x)=b(x)-\tilde{b}(x), \Sigma(x)=\frac{1}{2}\left(\sigma^{2}(x)-\tilde{\sigma}^{2}(x)\right), \text { and } U(t, x)=u(t, x)-\tilde{u}(t, x) .
$$

Then $U(t, x)$ satisfies

$$
\partial_{t} U=\frac{1}{2} \sigma^{2}(x) \partial_{x x} U+b(x) \partial_{x} U+B(x) \partial_{x} \tilde{u}+\Sigma(x) \partial_{x x} \tilde{u}, t \in[0, T), x \in \mathbb{R},
$$

and $U(0, x)=0$ for all $x \in \mathbb{R}$.

Let $x_{0} \in \omega$. We define:

$$
\begin{aligned}
& x_{B}^{*}=\sup \left\{x>x_{0} \text { such that } B \equiv 0 \text { on }\left[x_{0}, x\right]\right\}, \\
& x_{\Sigma}^{*}=\sup \left\{x>x_{0} \text { such that } \Sigma \equiv 0 \text { on }\left[x_{0}, x\right]\right\} .
\end{aligned}
$$

Then, four cases may occur.

Case 1: we assume that $x_{B}^{*}<x_{\Sigma}^{*}$. Using the piecewise analyticity of $B$, and from the definitions of $x_{B}^{*}$, we obtain the existence of some $x_{2} \in\left(x_{B}^{*}, x_{\Sigma}^{*}\right)$ such that $B(x) \neq 0$ for all $x \in\left(x_{B}^{*}, x_{2}\right]$, i.e., $B$ has a constant strict sign in $\left(x_{B}^{*}, x_{2}\right]$. Moreover, $\Sigma(x)=0$ for all $x \in\left(x_{B}^{*}, x_{2}\right]$, thus $U$ satisfies:

$$
\partial_{t} U-\mathscr{L} U=B(x) \partial_{x} \tilde{u}, t \in[0, T), x \in\left(x_{0}, x_{2}\right),
$$

where $\mathscr{L} U:=\frac{1}{2} \sigma^{2}(x) \partial_{x x} U+b(x) \partial_{x} U$. Take $k=1$ in the definition of $f(s)=s^{k}$. We have $\partial_{x} \tilde{u}(0, x)=f^{\prime}(x)=1$ for all $x \in \mathbb{R}$, which implies that there exists $\varepsilon^{\prime} \in$ $(0, \varepsilon)$ such that $\partial_{x} \tilde{u}(t, x)$ is positive on $\left[0, \varepsilon^{\prime}\right) \times\left[x_{0}, x_{2}\right]$. Finally, the term $B(x) \partial_{x} \tilde{u}$ in the right hand side of (18) has a constant sign in $\left(0, \varepsilon^{\prime}\right) \times\left[x_{0}, x_{2}\right]$. Without loss of generality, we can assume that:

$$
B(x) \partial_{x} \tilde{u} \geq 0 \text { for }(t, x) \text { in }\left[0, \varepsilon^{\prime}\right) \times\left[x_{0}, x_{2}\right] .
$$


We then observe that $\partial_{t} U\left(0, x_{2}\right)=B\left(x_{2}\right) \geq 0$ and, from the definition of $x_{2}$, the inequality is strict: $\partial_{t} U\left(0, x_{2}\right)>0$. Thus, (even if it means reducing $\varepsilon^{\prime}>0$ ),

$$
U\left(t, x_{2}\right)>0 \text { for } t \in\left(0, \varepsilon^{\prime}\right) .
$$

Finally, $U$ satisfies

$$
\left\{\begin{array}{l}
\partial_{t} U-\mathscr{L} U \geq 0, t \in\left(0, \varepsilon^{\prime}\right), x \in\left(x_{0}, x_{2}\right) \\
U\left(t, x_{0}\right)=0, U\left(t, x_{2}\right)>0, t \in\left(0, \varepsilon^{\prime}\right) \\
U(0, x)=0, x \in\left(x_{0}, x_{2}\right)
\end{array}\right.
$$

From strong parabolic maximum principle $U(t, x)>0$ in $\left(0, \varepsilon^{\prime}\right) \times\left(x_{0}, x_{2}\right)$. This contradicts the assumption $\mathscr{O}_{1}\left[X_{t}\right]=\mathscr{O}_{1}\left[\tilde{X}_{t}\right]$ of Theorem 1. Thus, Case 1 is ruled out.

Case 2: we assume that $x_{B}^{*}>x_{\Sigma}^{*}$. With the same type of arguments as in Case 1, we obtain the existence of some $x_{2} \in\left(x_{\Sigma}^{*}, x_{B}^{*}\right)$ such that $\Sigma(x) \neq 0$ for all $x \in\left(x_{\Sigma}^{*}, x_{2}\right]$, i.e., $\Sigma$ has a constant strict sign in $\left(x_{\Sigma}^{*}, x_{2}\right]$. Moreover, $B(x)=0$ for all $x \in\left(x_{\Sigma}^{*}, x_{2}\right]$, thus $U$ satisfies:

$$
\partial_{t} U-\mathscr{L} U=\Sigma(x) \partial_{x x} \tilde{u}, t \in[0, T), x \in\left(x_{0}, x_{2}\right) .
$$

Take $k=2$ in the definition of $f(s)=s^{k}$. We have $\partial_{x x} \tilde{u}(0, x)=f^{\prime \prime}(x)=2$ for all $x \in \mathbb{R}$. Thus, with the same arguments as in Case 1 , we get:

$$
\Sigma(x) \partial_{x x} \tilde{u} \geq 0 \text { for }(t, x) \text { in }\left[0, \varepsilon^{\prime}\right) \times\left[x_{0}, x_{2}\right]
$$

and $\partial_{t} U\left(0, x_{2}\right)>0$. Thus, $U$ again satisfies (21), and the strong parabolic maximum principle implies $U(t, x)>0$ in $\left(0, \varepsilon^{\prime}\right) \times\left(x_{0}, x_{2}\right)$, leading to a contradiction with the assumption $\mathscr{O}_{2}\left[X_{t}\right]=\mathscr{O}_{2}\left[\tilde{X}_{t}\right]$ of Theorem 1 . Thus, Case 2 is ruled out.

Case 3: we assume that $x_{B}^{*}=x_{\Sigma}^{*}<+\infty$. Let us set

$$
G(t, x)=B(x) \partial_{x} \tilde{u}+\Sigma(x) \partial_{x x} \tilde{u},
$$

corresponding to the right-hand side in (16). Then, set

$$
l^{*}=\lim _{x \rightarrow x_{B}^{*}, x>x_{B}^{*}} \frac{\Sigma(x)}{B(x)} .
$$

From the analyticity of $\Sigma$ and $B$ in a right neighborhood of $x_{B}^{*}, l^{*}$ is well-defined and only two situations may occur: either $\left|l^{*}\right|<+\infty$ or $\left|l^{*}\right|=+\infty$.

Assume first that $\left|l^{*}\right|<+\infty$. Take $k=1$ in the definition of $f(s)=s^{k}$. Thus, 


$$
\partial_{x} \tilde{u}(0, x)=1 \text { and } \partial_{x x} \tilde{u}(0, x)=0 .
$$

Let $x_{2}>x_{B}^{*}$ such that $B(x) \neq 0$ in $\left(x_{B}^{*}, x_{2}\right]$ and $\Sigma(x) / B(x)$ remains bounded in $\left(x_{B}^{*}, x_{2}\right]$. Without loss of generality, we can assume that $B>0$ in $\left(x_{B}^{*}, x_{2}\right]$. Using (24), and since $\left|l^{*}\right|<+\infty$, we obtain the existence of $\varepsilon^{\prime} \in(0, \varepsilon)$ such that

$$
\frac{G(t, x)}{B(x)}=\partial_{x} \tilde{u}+\frac{\Sigma(x)}{B(x)} \partial_{x x} \tilde{u}>0 \text { for }(t, x) \text { in }\left(0, \varepsilon^{\prime}\right) \times\left(x_{0}, x_{2}\right),
$$

and $G(t, x)$ satisfies the same inequality. Thus, again, $U$ satisfies (21), and the strong parabolic maximum principle implies that $U(t, x)>0$ in $\left(0, \varepsilon^{\prime}\right) \times\left(x_{0}, x_{2}\right)$ and a contradiction with the assumption $\mathscr{O}_{1}\left[X_{t}\right]=\mathscr{O}_{1}\left[\tilde{X}_{t}\right]$ of Theorem 1 . The assumption $\left|l^{*}\right|<+\infty$ is then ruled out.

Assume now that $\left|l^{*}\right|=+\infty$. Take $k=2$ in the definition of $f(s)=s^{k}$. This time,

$$
\partial_{x} \tilde{u}(0, x)=2 x \text { and } \partial_{x x} \tilde{u}(0, x)=2 .
$$

Let $x_{2}>x_{\Sigma}^{*}$ such that

$$
\Sigma(x) \neq 0 \text { and }|2 x(B(x) / \Sigma(x))|<1 \text { in }\left(x_{\Sigma}^{*}, x_{2}\right] .
$$

Without loss of generality, we assume that $\Sigma>0$ in $\left(x_{\Sigma}^{*}, x_{2}\right]$. Using (25), and since $\left|l^{*}\right|=+\infty$, we can define $\varepsilon^{\prime} \in(0, \varepsilon)$ such that

$$
\frac{G(t, x)}{\Sigma(x)}=\frac{B(x)}{\Sigma(x)} \partial_{x} \tilde{u}+\partial_{x x} \tilde{u}>0 \text { for }(t, x) \text { in }\left(0, \varepsilon^{\prime}\right) \times\left(x_{0}, x_{2}\right) .
$$

Again, using the strong parabolic maximum principle, we get a contradiction with the assumption $\mathscr{O}_{2}\left[X_{t}\right]=\mathscr{O}_{2}\left[\tilde{X}_{t}\right]$ of Theorem 1. Case 3 is then ruled out.

Finally, as Cases 1,2, 3 are ruled out, we necessarily have $x_{B}^{*}=x_{\Sigma}^{*}=+\infty$, which show that $B \equiv \Sigma \equiv 0$ in $\left(x_{0},+\infty\right)$. Using the same arguments with $\left(x_{B}^{*}\right)^{-}=\inf \{x<$ $x_{0}$ such that $B \equiv 0$ on $\left.\left[x, x_{0}\right]\right\}$ and $\left(x_{\Sigma}^{*}\right)^{-}=\inf \left\{x<x_{0}\right.$ such that $\Sigma \equiv 0$ on $\left.\left[x, x_{0}\right]\right\}$, instead of $x_{B}^{*}$ and $x_{\Sigma}^{*}$, we also check that $B \equiv 0$ in $B \equiv \Sigma \equiv 0$ in $\left(-\infty, x_{0}\right)$ and consequently $B \equiv \Sigma \equiv 0$ in $\mathbb{R}$ which concludes the proof of Theorem 1 .

\section{References}

1. S Albeverio, Ph Blanchard, S Kusuoka, and L Streit. An inverse problem for stochastic differential equations. Journal of statistical physics, 57(1,2), 1989.

2. G Bao, C C Chen, , and P Li. Inverse random source scattering problems in several dimensions,. SIAM/ASA J. Uncertaint. Quantif, 4(1):1263-1287, 2016. 
3. M Belassoued and M Yamamoto. Inverse source problem for a transmission problem for a parabolic equation. Journal of Inverse and Ill-Posed Problems, 14(1):47-56, 2006.

4. A L Bukhgeim and M V Klibanov. Uniqueness in the large of a class of multidimensional inverse problems. Soviet Mathematics - Doklady, 24:244-247, 1981.

5. M Choulli and M Yamamoto. Uniqueness and stability in determining the heat radiative coefficient, the initial temperature and a boundary coefficient in a parabolic equation. Nonlinear Analysis: Theory, Methods \& Applications, 69(11):3983-3998, 2008.

6. M Cristofol, P Gaitan, K Niinimaki, and O Poisson. Inverse problem fora coupled parabolic system with discontinuous conductivities: one dimensional case. Inverse problems and imaging, 7:159-182, 2013.

7. M Cristofol, J Garnier, F Hamel, and L Roques. Uniqueness from pointwise observations in a multi-parameter inverse problem. Commun Pur Appl Anal, 11:1-15, 2011.

8. M Cristofol, I Kaddouri, G Nadin, and L Roques. Coefficient determination via asymptotic spreading speeds. Inverse Problems, 30(3):035005, 2014.

9. M Cristofol, $\mathrm{S} \mathrm{Li}$, and E Soccorsi. Determining the waveguide conductivity in a hyperbolic equation from a single measurement on the lateral boundary , 6, (3), 2016. Journal of mathematical control and related fields, 3:407-427, 2016.

10. M Cristofol and L Roques. Biological invasions: Deriving the regions at risk from partial measurements. Math Biosci, 215(2):158-166, 2008.

11. M Cristofol and L Roques. Stable estimation of two coefficients in a nonlinear fisher-kpp equation. Inverse Problems, 29(9):095007, 2013.

12. M Cristofol and L Roques. Simultaneous determination of the drift and diffusion coefficients in stochastic differential equations. Inverse Problems, 33:095006, 2017.

13. M S Hussein and D Leisnic. Simultaneous determination of time and space-dependent coefficients in a parabolic equation. Commun. Nonlinear. Sci. Numer. Simul., 33:194-217, 2016.

14. O Y Immanuvilov and M Yamamoto. Lipschitz stability in inverse parabolic problems by the Carleman estimate. Inverse Problems, 14:1229-1245, 1998.

15. S Kirkpatrick, C D Gelatt, and M P Vecchi. Optimization by simulated annealing. Science, 220:671-680, 1983.

16. $\mathrm{P} \mathrm{J} \mathrm{Li.} \mathrm{An} \mathrm{inverse} \mathrm{random} \mathrm{source} \mathrm{scattering} \mathrm{problem} \mathrm{in} \mathrm{inhomogeneous} \mathrm{media,.} \mathrm{Inverse}$ Problems, 27(3):035004, 2011.

17. $\mathrm{P}$ Niu, $\mathrm{S} \mathrm{Lu}$, and $\mathrm{J}$ Cheng. On periodic parameter identification in stichastics differential equation. Inverse problems and imaging, 13:513-543, 2019.

18. B Øksendal. Stochastic differential equations. Springer, 2003.

19. L Roques, M D Chekroun, M Cristofol, S Soubeyrand, and M Ghil. Parameter estimation for energy balance models with memory. Proc R Soc A, 470(2169):20140349, 2014.

20. L Roques and M Cristofol. On the determination of the nonlinearity from localized measurements in a reaction-diffusion equation. Nonlinearity, 23:675-686, 2010.

21. L Roques and M Cristofol. The inverse problem of determining several coefficients in a nonlinear Lotka-Volterra system. Inverse Problems, 28:075007, 2012.

22. H Sorensen. Parametric inference for diusion processes observed at discrete points in time: A survey,. International Statistical Review, 72:337-354, 2003.

23. M Yamamoto. Carleman estimates for parabolic equations and applications. Inverse Problems, 25(12):123013, 2009.

24. M Yamamoto and J Zou. Simultaneous reconstruction of the initial temperature and heat radiative coefficient. Inverse Problems, 17:1181-1202, 2001. 